\title{
Self-similarities of periodic structures for a discrete model of a two-gene system
}

\author{
S.L.T. de Souza ${ }^{\mathrm{a}, *}$, A.A. Lima ${ }^{\mathrm{b}}$, I.L. Caldas ${ }^{\mathrm{c}}$, R.O. Medrano-T. ${ }^{\mathrm{d}}$, Z.O. Guimarães-Filho $^{\mathrm{e}}$ \\ a Departamento de Física e Matemática, Universidade Federal de São João del-Rei, Ouro Branco, MG, Brazil \\ b Escola de Farmácia, Universidade Federal de Ouro Preto, Ouro Preto, MG, Brazil \\ c Instituto de Física, Universidade de São Paulo, São Paulo, SP, Brazil \\ d Departamento de Ciências Exatas e da Terra, Universidade Federal de São Paulo, Diadema, SP, Brazil \\ e Aix-Marseille Univ., CNRS PIIM UMR6633, International Institute for Fusion Science, Marseille, France
}

\section{A R T I C L E I N F O}

\section{Article history:}

Received 10 December 2011

Accepted 15 February 2012

Available online 21 February 2012

Communicated by A.R. Bishop

\section{Keywords:}

Arnold tongues

Shrimps

Golden ratio

Lyapunov exponents

Two-gene model

\begin{abstract}
A B S T R A C T
We report self-similar properties of periodic structures remarkably organized in the two-parameter space for a two-gene system, described by two-dimensional symmetric map. The map consists of difference equations derived from the chemical reactions for gene expression and regulation. We characterize the system by using Lyapunov exponents and isoperiodic diagrams identifying periodic windows, denominated Arnold tongues and shrimp-shaped structures. Period-adding sequences are observed for both periodic windows. We also identify Fibonacci-type series and Golden ratio for Arnold tongues, and period multiple-of-three windows for shrimps.
\end{abstract}

(c) 2012 Elsevier B.V. All rights reserved.

\section{Introduction}

In the last 20 years, a considerable amount of work has been done on nonlinear systems analyzing bifurcations in twodimensional parameter spaces [1-6]. As a result, the existence of regular orbits can be properly visualized on continuous sets of parameter spaces, generating periodic islands (windows) embedded into quasi-periodic or chaotic regions. Thus, noticeable periodic windows, such as Arnold tongues and shrimps, have been identified both for discrete-time and continuous-time systems [7-12].

In general, the periodic window distributions appear highly organized in parameter space. Recently, a striking spiral organization of shrimps has received significant attention [13-17]. The global organization of these periodic regions is composed of an infinite hierarchy of shrimps with different periods continuously connected in a spiral sequence. As another example of interesting result for periodic windows, Medeiros and co-workers have shown that weak periodic perturbations used to control chaos provoke replications of shrimps $[18,19]$.

As is well known, nonlinear dynamics approaches have been used extensively to study different types of systems, including

\footnotetext{
* Corresponding author.

E-mail address: thomaz@ufsj.edu.br (S.L.T. de Souza).
}

plasma physics [20], impact oscillators [21], dripping faucets [22], circadian rhythms [23], heart rhythms [24], and peroxidaseoxidase reactions [25], just to mention a few. In the biochemistry context, nonlinear mathematical models are generally derived from the chemical reactions, like the two-gene model proposed by Andrecut and Kauffman [26]. In this case, the chemical reactions correspond to gene expression and regulation. The dynamics are obtained from a two-dimensional map, whose discrete dynamical variables describe the evolution of the concentration levels of transcription factor proteins.

In this Letter, we provide numerical analysis in the parameter space for the two-gene system. Numerical studies for this system have shown a rich dynamical behavior with several nonlinear phenomena, such as quasi-periodic attractors, chaotic regimes, crises, and coexistence of attractors. As examples of periodic windows in two-dimensional parameter space, we identify Arnold tongues and shrimps. To characterize the possible solutions (attractors) we evaluate the Lyapunov exponents. In addition, we use isoperiodic diagrams to investigate the self-similarities commonly observed in these periodic structures. Our main purpose in this study is to shed further light on the topic of periodic windows characterization.

This Letter is organized as follows. In Section 2 we present the mathematical model used to study dynamics of the two-gene system. In Section 3, we examine self-similarities associated with periodic windows identified in the two-parameter space. The last section contains our main remarks. 
(a)
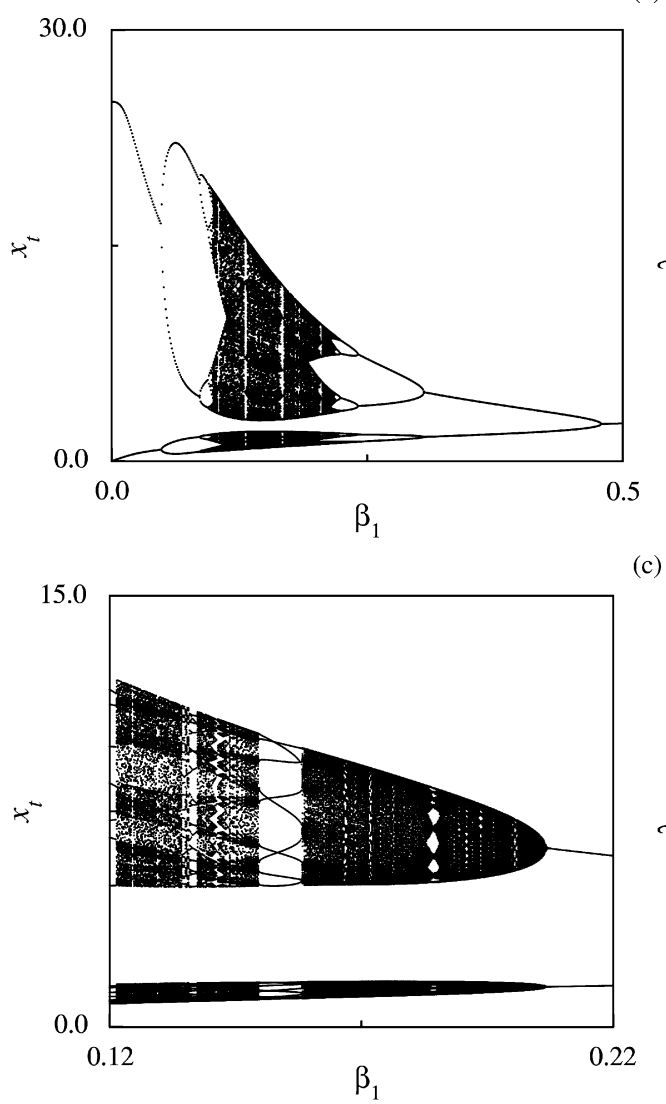

(c) (b)
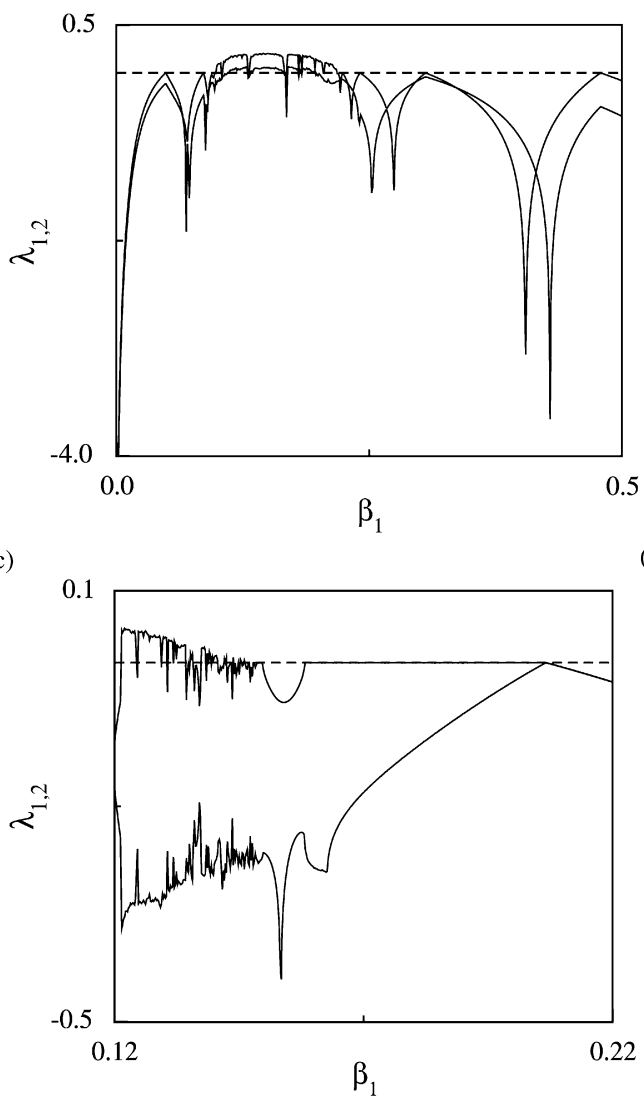

(d)

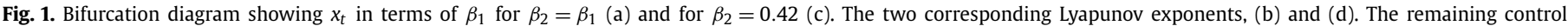
parameters are fixed at $\alpha=25.0, \varepsilon=0.1$, and $n=3$.

\section{Andrecut-Kauffman map}

For our numerical analysis, we consider a two-dimensional map proposed by Andrecut and Kauffman [26]. The map was used to investigate the dynamics of two-gene model for chemical reactions corresponding to gene expression and regulation. The discrete dynamical variables, denoted by $x_{t}$ and $y_{t}$, describe the evolutions of the concentration levels of transcription factor proteins. The map is given by the following difference equations:

$x_{t+1}=\frac{\alpha}{1+(1-\varepsilon) x_{t}^{n}+\varepsilon y_{t}^{n}}+\beta_{1} x_{t}$,

$y_{t+1}=\frac{\alpha}{1+(1-\varepsilon) y_{t}^{n}+\varepsilon x_{t}^{n}}+\beta_{2} y_{t}$.

As can be noted, the difference equations are obtained by coupling, with parameter $\varepsilon$, two single (one-dimensional) maps. For the values of the control parameters, the following ranges can be used $\alpha \in[0,100], \beta_{1} \in[0,1), \beta_{2} \in[0,1), n=1,2,3,4$, and $\varepsilon \in[0,1]$.

In order to calculate the Lyapunov exponents [27], we evaluate $\lambda_{i}=\lim _{t \rightarrow \infty}(1 / t) \ln \left|\Lambda_{i}(t)\right|(i=1,2)$, where $\Lambda_{i}(t)$ are the eigenvalues of the matrix $A=J_{1} \cdot J_{2}, \ldots, J_{t}$, with the Jacobian matrix, $J_{t}$, computed at time $t$.

\section{Arnold tongues and shrimp-shaped structures}

The dynamics was investigated using bifurcation diagrams, Lyapunov exponents, parameter plane diagrams, and isoperiodic diagrams. We consider the control parameters $\beta_{1}$ and $\beta_{2}$ for our simulations. The remaining parameters are fixed at $\alpha=25.0, \varepsilon=0.1$, and $n=3$. Furthermore, the simulations are performed with the initial conditions fixed at $\left(x_{0}, y_{0}\right)=(0.5,0.5)$.

First of all, to obtain a representative example of the kind of dynamics could be generated by the two-gene model, we use bifurcation diagrams identifying the possible solutions (attractors) in terms of one-dimensional parameter with fixed initial conditions. This diagram is constructed varying one control parameter. For each value of the parameter, we neglect a sufficiently large number of iterations to eliminate the transient behavior, then we plot the subsequent points of the dynamical variable $x_{t}$. To characterize the nature of the attractors obtained, we evaluate the Lyapunov exponents. The positive largest Lyapunov exponent (LLE) for maps indicates a chaotic attractor, the negative a periodic, and the zero a quasi-periodic (or a bifurcation point).

In Fig. 1(a) for $\beta_{1}=\beta_{2}$, we plot a bifurcation diagram showing the asymptotic values of $x_{t}$ as a function of $\beta_{1}$. The corresponding Lyapunov exponents are shown in Fig. 1(b). In this case, as can be seen, the bifurcation diagram is composed of a periodbubbling scenario with chaotic region (positive LLE) interrupted by periodic windows (negative LLE). One of the Lyapunov exponents is zero at the bifurcation points. In addition, there are solutions with two positive Lyapunov exponents for a small range of the parameter. (This kind of solution is commonly observed for systems composed of one-dimensional coupled maps.) For the parameter value $\beta_{2}=0.42$, we also identify in terms of $\beta_{1}$, as shown in Figs. 1(c)-(d), chaotic and quasi-periodic (zero LLE) attractors also interrupted by periodic windows (negative LLE).

As a result of the preliminary numerical analysis, we verify here that the two-gene model presents a plethora of complex dynamical phenomena. In order to obtain a better understanding of the rich dynamics involved and associated with periodic windows, 

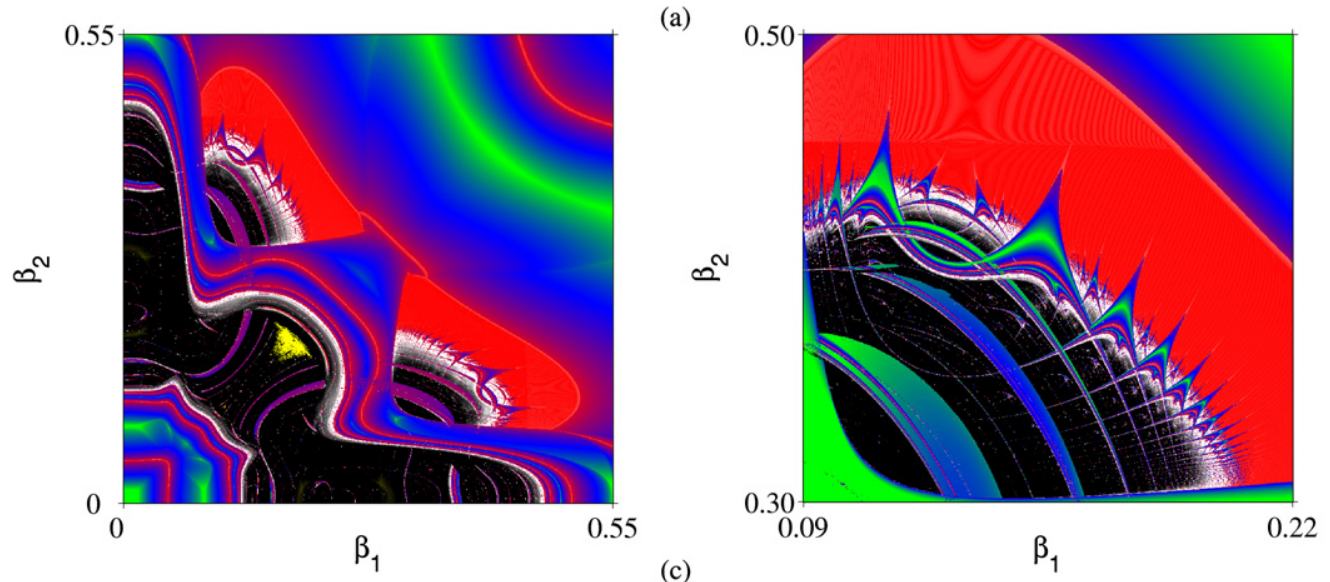

(b)
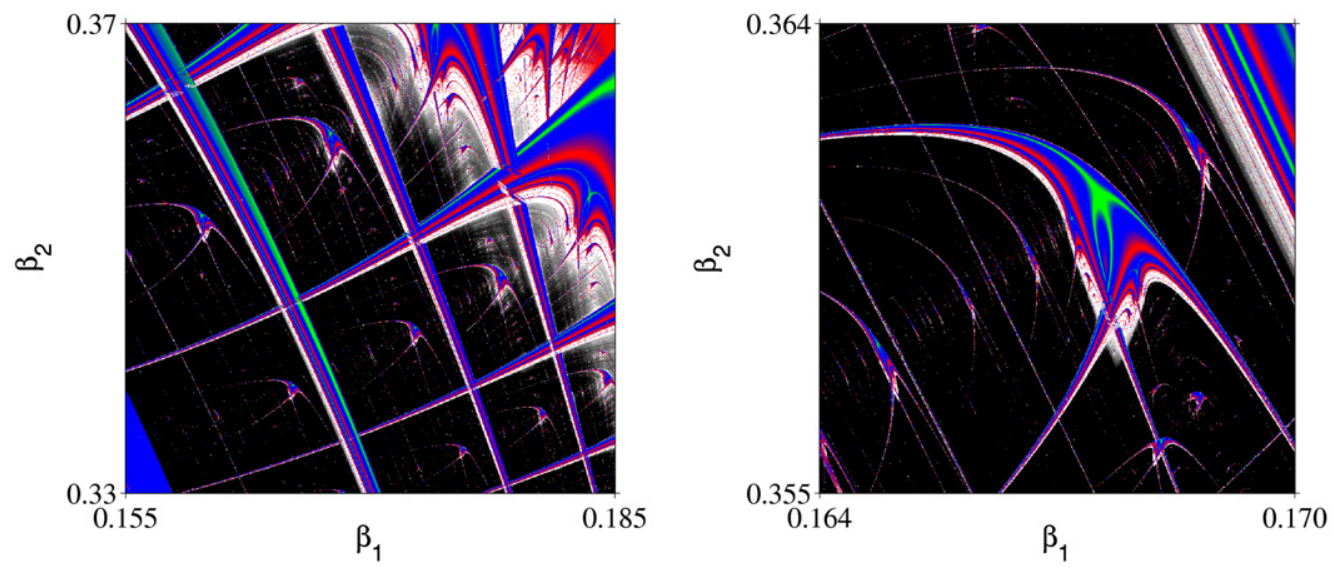

(d)

Fig. 2. (Color online.) (a) Parameter plane diagram for $\beta_{2}$ versus $\beta_{1}$. (b)-(d) Successive magnifications.

it is indispensable to investigate the model in the parameter space. For that reason, we construct parameter plane diagrams, in terms of $\beta_{1}$ and $\beta_{2}$, using a grid of $800 \times 800$ cells. The color of each cell is allocated according to the Lyapunov exponents.

In Figs. 2(a)-(d), we present a parameter plane diagram and successive magnification views of the small box in the previous figures (color online). Periodic solutions are plotted in blue and green scale ranges (bifurcation points in red), quasi-periodic in red, and chaotic in white and black. In yellow are plotted solutions with two positive Lyapunov exponents. The limit range of the color scales is redefined, for each diagram, according to the Lyapunov exponent values.

As can be seen in Fig. 2(a), the periodic regions are symmetrically organized in the parameter plane. (This fact was expected since the considered map, when exchanging the values of $\beta_{1}$ and $\beta_{2}$, is invariant by a change of variables $x$ and $y$.) In Fig. 2(b), we present the magnification of one of the two areas with quasi-periodic solutions (in red) and resonance regions (in blue or green), so-called Arnold tongues. In Figs. 2(c)-(d), we provide magnifications of rectangular area revealing shrimpshaped periodic regions. (The shrimps, term coined by Gallas [7], are composed of the central body bordered by a saddle-node and a flip bifurcations.) Thus, we identify in Figs. 2(a)-(d) two kinds of periodic structures (Arnold tongues and shrimps). Both periodic structures have self-similar properties. In other words, we can observe the repetition of structures for different length scales.

For further analysis of the self-similar organization of the Arnold tongues, in Figs. 3(a)-(d) we consider isoperiodic diagrams constructed with a grid of $800 \times 800$ cells. For each cell, we evaluate the period of the attractors, whose values are plotted using a grayscale. Initially, in Fig. 3(a) we identify a period-adding se- quence for the tongues (plotted in black) with accumulation on period-4 region (in dark gray). The period of the sequence increases by $4(18 \rightarrow 22 \rightarrow 26 \rightarrow 30 \rightarrow 34 \ldots)$, which is the same period of the accumulation structure. Similar feature was reported for shrimps by Bonatto and co-workers [28].

In addition to period-adding sequences, we now examine the periodicities of the other tongues. In Fig. 3(b), we provide a magnification of a region showing structures between tongues with periods 18 and 22 (in dark gray). The periodicity of the largest tongue (in black) is 40 , i.e. the sum of 18 and 22 . Between regions with periods 22 and 40, we identify one with period 62 . Thus, the generated sequence, specifying the periods, is composed of numbers: $18 \rightarrow 22 \rightarrow 40 \rightarrow 62 \rightarrow 102 \ldots$. As in the well-known Fibonacci sequence, each new term of the sequence is the sum of two previous ones. Not surprisingly, but it is interesting to note, as shown in Table 1, that the ratios of consecutive terms of the periodic value sequences gradually converge to the Golden ratio ( $\varphi \equiv \frac{1+\sqrt{5}}{2} \approx 1.618033989$ ) [29], also called the Divine Proportion.

In Fig. 3(c), we show one more example of the period-adding sequence for the tongues (in black). In this case, the sequence increases by $22(40 \rightarrow 62 \rightarrow 84 \rightarrow 106 \ldots)$, that is the period of the accumulation region (in dark gray). Between tongues with periods 40 and 62 (dark gray), in Fig. 3(d) we verify a period-102 tongue (black), that is generated obeying again the simple rule with the sum of two previous values.

In the same way by using isoperiodic diagrams, we investigate the self-similar properties of the shrimp-shaped structures. First, in Fig. 4 we can see some series, remarkably organized, of the period-adding sequences. Furthermore, from this figure we can realize several periodic structures crossing each other. For these regions, coexistence of attractors with fractal basin boundaries, 


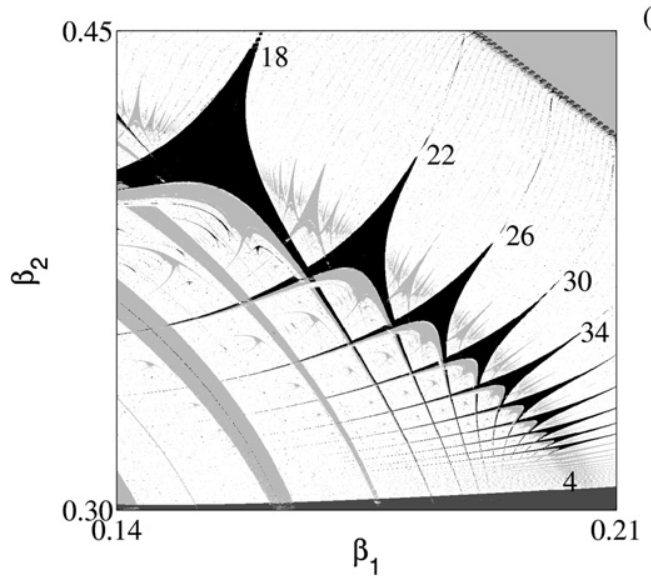

(a)

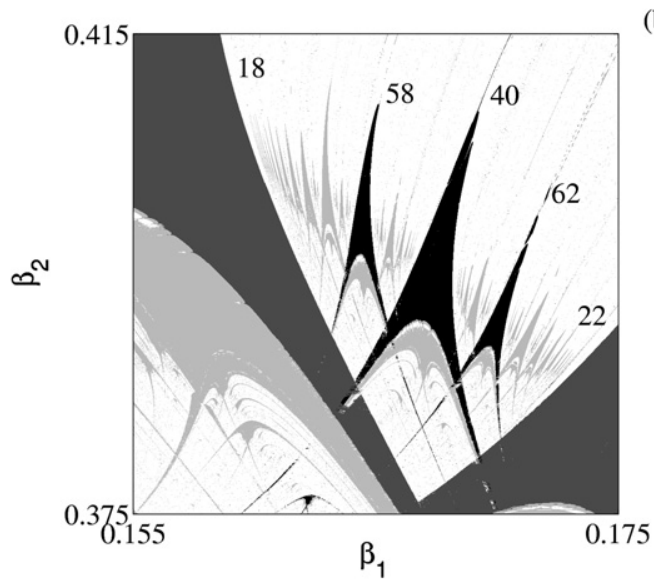

(b)

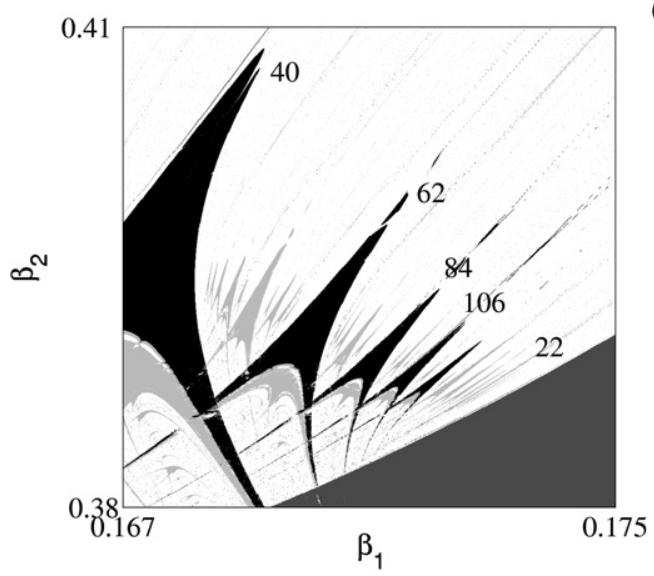

(c)

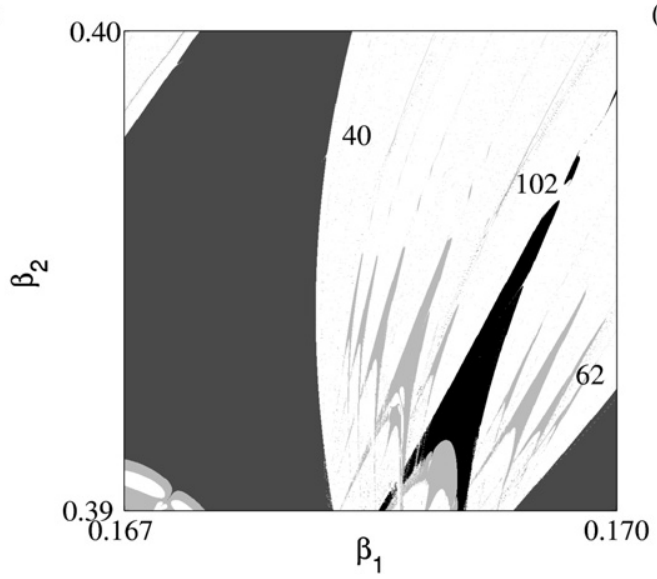

(d)

Fig. 3. (a) Isoperiodic diagram of the parameters $\beta_{2}$ versus $\beta_{1}$ showing the organization of Arnold tongues. (b)-(d) Successive magnifications.

Table 1

Convergence to Golden ratio $\varphi \equiv \frac{1+\sqrt{5}}{2} \approx 1.618033989$.

\begin{tabular}{rll}
\hline$i$ & $P_{i+1} / P_{i}$ & $\varphi_{i}$ \\
\hline 0 & $22 / 18$ & 1.222222222 \\
1 & $40 / 22$ & 1.818181818 \\
2 & $62 / 40$ & 1.550000000 \\
3 & $102 / 62$ & 1.645161290 \\
4 & $164 / 102$ & 1.607843137 \\
5 & $266 / 164$ & 1.621951220 \\
10 & $2948 / 1822$ & 1.618002195 \\
15 & $32694 / 20206$ & 1.618034247 \\
20 & $362582 / 224088$ & 1.618033987 \\
25 & $4021096 / 2485174$ & 1.618033989 \\
\hline
\end{tabular}

not shown in this work, are identified. (The addition of parametric noise can cause basin hopping, i.e. the alternate switching among different coexisting attractors. Depending on the amount of noise, the system oscillates chaotically [30].)

Now, we explore the manifestations of self-similarity associated with a shrimp. For that purpose, we evaluate the periodicity of the secondary shrimps observed close the legs of a main shrimp. In Fig. 5(a), we plot a period-40 shrimp (dark gray) with the secondary ones (in black). The largest secondary shrimp, as shown in Fig. 5(b), presents a period 120 (40 multiply by 3 ). It is interesting to note the presence of the signature of chaos with period-3 windows [31], or more exactly, period multiple of 3. Moreover, from this figure we identify again a period-adding sequence associated with the period of accumulation region, i.e. the sequence is increasing, by 40 , with same period of the main shrimp. Finally, we provide in Figs. 5(c) and (d) one more example of the same selfsimilarity characteristics for a period-44 shrimp.

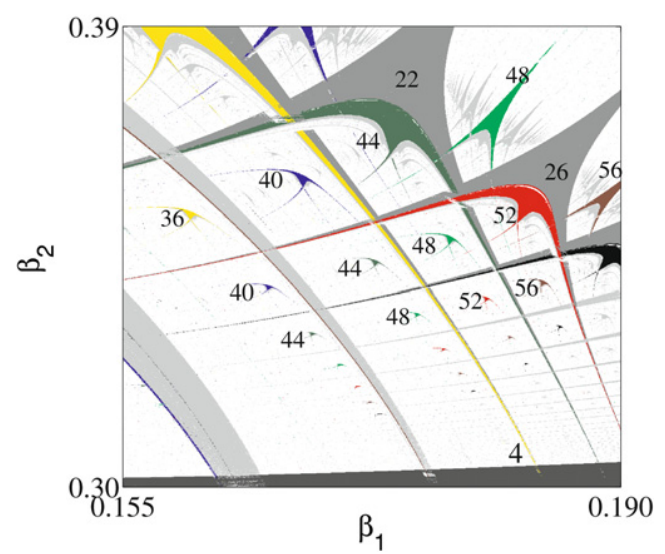

Fig. 4. (Color online.) Isoperiodic diagram of the parameters $\beta_{2}$ versus $\beta_{1}$ showing period-adding sequences of the shrimps.

\section{Final remarks}

We have characterized self-similar properties of Arnold tongues and shrimp-shaped structures (periodic windows) observed in two-parameter space for the two-gene model. We identified period-adding sequences with accumulation horizons for both periodic windows. The sequences increase by the same values of period evaluated in the accumulation regions. Furthermore, we determined the period of the Arnold tongues recognizing a Fibonacci-type sequence, i.e. the sequence generated by the same recurrence rule of the Fibonacci series but with other initial terms. This sequence is also associated with Golden ratio. In the end, 
(a)

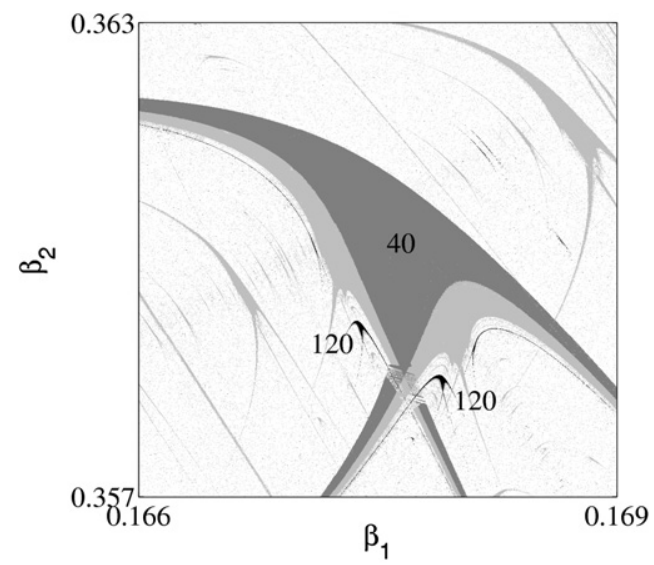

(c)

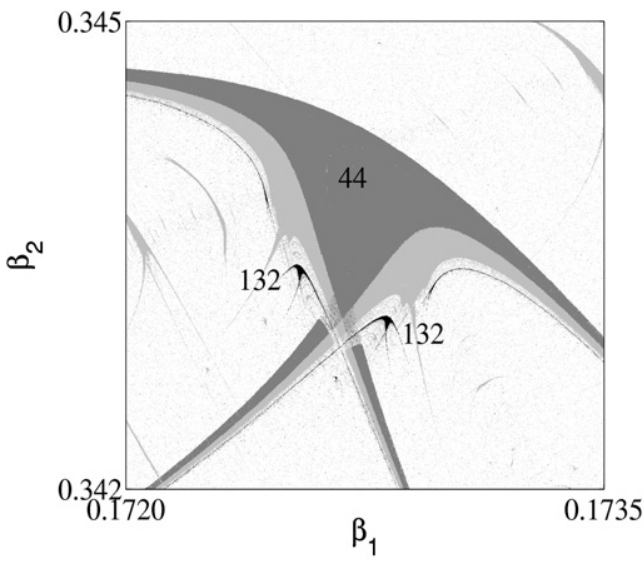

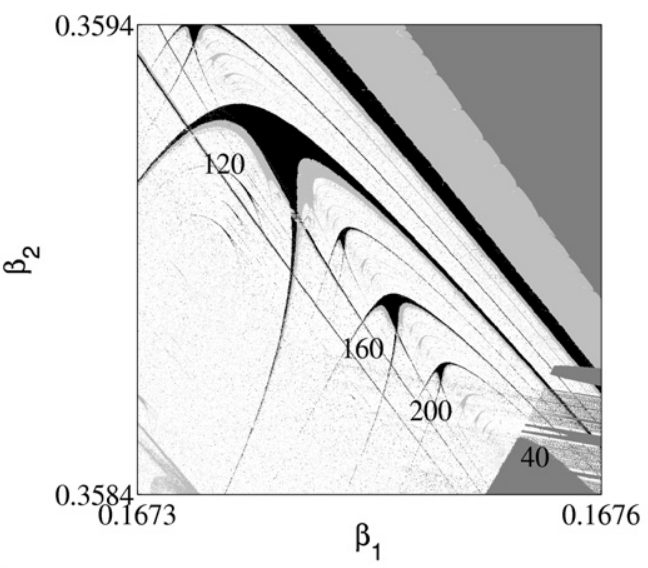

(b)

(d)

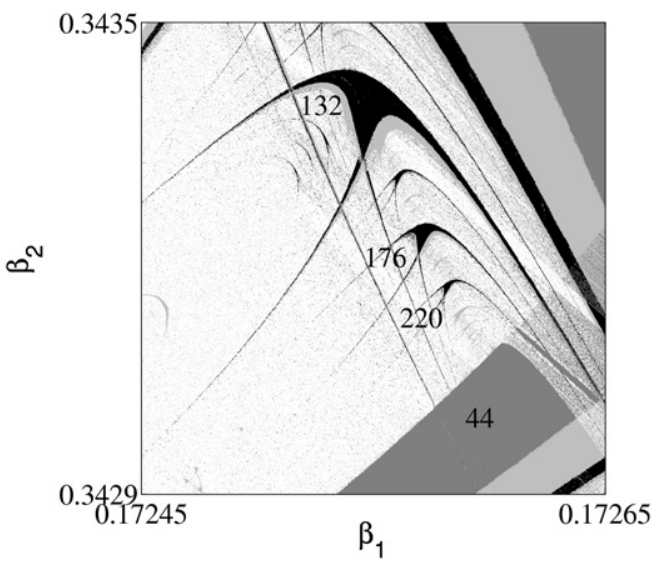

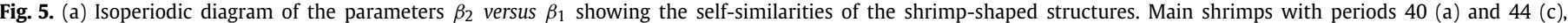
and magnifications of their corresponding secondary shrimps (b) and (d).

we explored self-similar features of the shrimps comparing their periodicities with the largest secondary shrimps (observed close the legs of a main shrimp). In this case, the period of the secondary shrimps are obtained multiplying by 3 the period of the corresponding main shrimp. Thus, we surprisingly verified the occurrence of a typical signature of nonlinear dynamics denominated, period-three implies chaos.

To conclude, we remark that the effect of noise perturbations was not considered in our present investigations. In general, the noise perturbations provoke basin hopping, i.e., the alternate switching among different coexisting attractors with fractal basin boundaries. Consequently, we expect that the addition of noise should modify significantly the dynamics of the two-gene system, altering or destroying the periodic windows. However, further numerical investigation should be carried out to better understand the noise effect in the considered system. In fact, we are currently working in this direction.

\section{Acknowledgements}

This work was made possible by partial financial support from the following Brazilian government agencies: FAPESP, CNPq, and CAPES.

\section{References}

[1] J.A.C. Gallas, Phys. Rev. Lett. 70 (1993) 2714.

[2] M.S. Baptista, I.L. Caldas, Chaos Solitons Fractals 7 (1996) 325.

[3] E. Barreto, B.R. Hunt, C. Grebogi, J.A. Yorke, Phys. Rev. Lett. 78 (1997) 4561
[4] S.L.T. de Souza, M. Wiercigroch, I.L. Caldas, J.M. Balthazar, Chaos Solitons Fractals 38 (2008) 864

[5] E.N. Lorenz, Physica D 237 (2008) 1689.

[6] J.C.D. Cardoso, H.A. Albuquerque, R.M. Rubinger, Phys. Lett. A 373 (2009) 2050

[7] J.A.C. Gallas, Physica A 202 (1994) 196

[8] M.S. Baptista, Ph.D. thesis, Universidade de São Paulo, 1996

[9] C. Bonatto, J.C. Garreau, J.A.C. Gallas, Phys. Rev. Lett. 95 (2005) 143905

[10] P.F.A. Di Donato, E.E.N. Macau, C. Grebogi, Nonlinear Dynam. 47 (2007) 75.

[11] S.L.T. de Souza, I.L. Caldas, R.L. Viana, Math. Probl. Eng. 2009 (2009) 290356.

[12] V. Maistrenko, A. Vasylenko, Y. Maistrenko, E. Mosekilde, Internat. J. Bifur. Chaos Appl. Sci. Engrg. 20 (2010) 1811.

[13] O. De Feo, G.M. Maggio, M.P. Kennedy, Internat. J. Bifur. Chaos 10 (2000) 935.

[14] C. Bonatto, J.A. Gallas, Phys. Rev. Lett. 101 (2008) 054101.

[15] J. Slipantschuk, E. Ullner, M.S. Baptista, M. Zeineddine, M. Thiel, Chaos 20 (2010) 045117.

[16] R. Stoop, P. Benner, Y. Uwate, Phys. Rev. Lett. 105 (2010) 074102.

[17] R.O. Medrano-T., I.L. Caldas, arXiv:1012.2241v1, 2010.

[18] E.S. Medeiros, S.L.T. de Souza, R.O. Medrano-T., I.L. Caldas, Phys. Lett. A 374 (2010) 2628.

[19] E.S. Medeiros, S.L.T. de Souza, R.O. Medrano-T., I.L. Caldas, Chaos Solitons Fractals 44 (2011) 982

[20] M. Roberto, E.C. da Silva, I.L. Caldas, R.L. Viana, Phys. Plasmas 11 (2004) 214

[21] J. Ing, E. Pavlovskaia, M. Wiercigroch, S. Banerjee, Physica D 239 (2010) 312.

[22] M.B. Reyes, R.D. Pinto, A. Tufaile, J.C. Sartorelli, Phys. Lett. A 300 (2002) 192.

[23] G.A. Oda, W.O. Friesen, J. Biol. Rhythms 17 (2002) 76.

[24] S.R.F.S.M. Gois, M.A. Savi, Chaos Solitons Fractals 41 (2009) 2553.

[25] A. Sensse, M. Hauser, M. Eiswirth, J. Chem. Phys. 125 (2006) 014901.

[26] M. Andrecut, S.A. Kauffman, Phys. Lett. A 367 (2007) 281.

[27] S.L.T. de Souza, I.L. Caldas, Chaos Solitons Fractals 19 (2004) 569.

[28] C. Bonatto, J.A.C. Gallas, Y. Ueda, Phys. Rev. E 77 (2008) 026217.

[29] T.W. Dixon, T. Gherghetta, B.G. Kenny, Chaos 6 (1996) 32.

[30] S.L.T. de Souza, I.L. Caldas, R.L. Viana, A.M. Batista, T. Kapitaniak, Chaos Solitons Fractals 26 (2005) 1523.

[31] T.Y. Li, J.A. Yorke, Amer. Math. Monthly 82 (1975) 985 